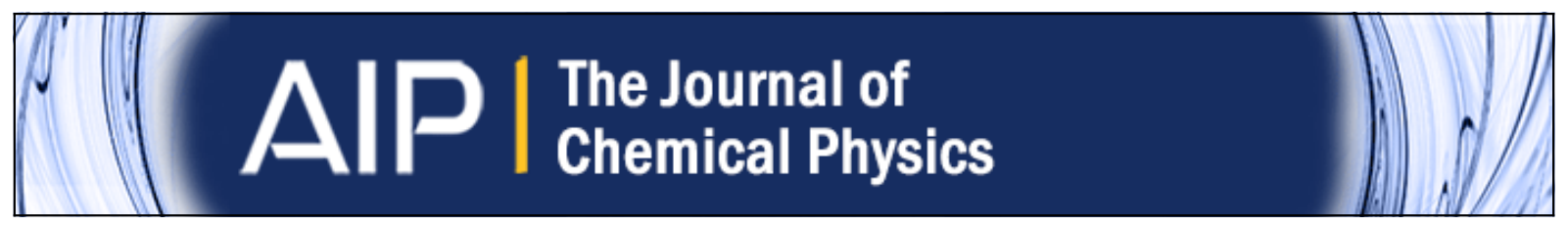

\title{
Single electron states in polyethylene
}

Y. Wang, D. MacKernan, D. Cubero, D. F. Coker, and N. Quirke

Citation: The Journal of Chemical Physics 140, 154902 (2014); doi: 10.1063/1.4869831

View online: http://dx.doi.org/10.1063/1.4869831

View Table of Contents: http://scitation.aip.org/content/aip/journal/jcp/140/15?ver=pdfcov

Published by the AIP Publishing

\section{Articles you may be interested in}

Atomic rearrangements in amorphous $\mathrm{Al} 2 \mathrm{O} 3$ under electron-beam irradiation

J. Appl. Phys. 113, 064312 (2013); 10.1063/1.4790705

Three-dimensional fabrication and characterisation of core-shell nano-columns using electron beam patterning of Ge-doped SiO2

Appl. Phys. Lett. 100, 263113 (2012); 10.1063/1.4731765

Nanometer scale study of $\mathrm{HfO} 2$ trap states using single electron tunneling force spectroscopy

Appl. Phys. Lett. 98, 172903 (2011); 10.1063/1.3580767

Surface plasmon density of states at the metal-dielectric interface: Dependence of metal layer thickness and dielectric material

J. Appl. Phys. 107, 014309 (2010); 10.1063/1.3275887

Electronic state modification in laser deposited amorphous carbon films by the inclusion of nitrogen

J. Appl. Phys. 104, 063701 (2008); 10.1063/1.2977718

How can you REACH $100 \%$

of researchers at the Top 100

Physical Sciences Universities?

With The Journal of Chemical Physics.

AIP $\left.\right|_{\text {Chemical Physics }} ^{\text {The Journal of }}$

THERE'S POWER IN NUMBERS. Reach the world with AIP Publishing.

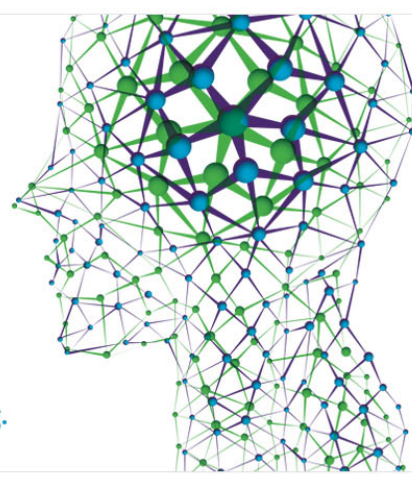




\title{
Single electron states in polyethylene
}

\author{
Y. Wang,,$^{1,2}$ D. MacKernan, ${ }^{2}$ D. Cubero, ${ }^{1,3, a)}$ D. F. Coker, ${ }^{2,4}$ and N. Quirke ${ }^{1,5, a)}$ \\ ${ }^{1}$ State Key Lab. of Electrical Insulation and Power Equipment, Xi'an Jiaotong University, No. 28 Xianning \\ West Road, Xi'an 710049, Shaanxi, China \\ ${ }^{2}$ School of Physics and Complex Adaptive Systems Laboratory, University College Dublin, Belfield, \\ Dublin 4, Ireland \\ ${ }^{3}$ Departmento de Física Aplicada I, Universidad de Sevilla, Calle Virgen de Africa 7, 41011 Seville, Spain \\ ${ }^{4}$ Department of Chemistry, Boston University, 590 Commonwealth Avenue, Boston, Massachusetts 02215, USA \\ ${ }^{5}$ Department of Chemistry, Imperial College, London SW7 2AY, United Kingdom
}

(Received 22 December 2013; accepted 11 March 2014; published online 15 April 2014)

\begin{abstract}
We report computer simulations of an excess electron in various structural motifs of polyethylene at room temperature, including lamellar and interfacial regions between amorphous and lamellae, as well as nanometre-sized voids. Electronic properties such as density of states, mobility edges, and mobilities are computed on the different phases using a block Lanczos algorithm. Our results suggest that the electronic density of states for a heterogeneous material can be approximated by summing the single phase density of states weighted by their corresponding volume fractions. Additionally, a quantitative connection between the localized states of the excess electron and the local atomic structure is presented. (C) 2014 AIP Publishing LLC. [http://dx.doi.org/10.1063/1.4869831]
\end{abstract}

\section{INTRODUCTION}

Electron trapping (carrier localisation) processes are of fundamental interest in the chemistry and physics of a wide range of technologies, for example, in photocatalysis, ${ }^{1}$ photovoltaics, ${ }^{2,3}$ organic thin film transistors (OTFTs), lightemitting diodes (OLEDs), ${ }^{4}$ and electrical insulation. ${ }^{5}$ More generally, such trapping can profoundly influence electron transfer processes that are fundamental to energy transfer in nature. ${ }^{6}$ Of particular interest, and our focus here, is the nature of trap states in polymeric materials. ${ }^{7}$ Polyethylene (PE) is the simplest organic insulator, playing a very important role in a number of power applications such as high-tension cable insulation. ${ }^{8}$ Despite a vast literature concerned with the experimental characterization of its electrical properties, very little is known about the details of the electrical behaviour of this material, and others like it, at the molecular level. An understanding of the mechanisms of charge trapping and transport in these insulators and how such processes are affected by local physical structures and chemical impurities could lead to the development of new materials. Such new materials could, for example, reduce energy loss in transmission over long distances and/or allow higher voltages to be used facilitating the development of smart grids and safe green energy transmission.

In recent years some progress has been made in understanding the fundamentals of charge trapping and transport in polyethylene using molecular modelling techniques. In previous work we identified physical (conformational) and chemical (impurities) traps in model alkane waxes thought to approximate the local structure of amorphous polyethylene and characterized them using $a b$ initio methods. ${ }^{9}$ The resulting

\footnotetext{
a) Authors to whom correspondence should be addressed. Electronic addresses: dcubero@us.es and n.quirke@imperial.ac.uk
}

density of states was employed in a Monte Carlo simulation to predict the current-voltage characteristics of a model of polyethylene. ${ }^{10}$ A more complete treatment of conformational trapping using a fast Fourier transform block Lanczos diagonalization algorithm allowed us to study the electronic states and mobility of excess electrons in a model of amorphous polyethylene using a novel pseudo potential for electron-PE interactions developed by fitting to conduction band energy data for fluid alkanes.

From our previous simulations, ${ }^{11}$ while the electronic states in fluid methane are extended throughout the whole finite size simulation sample, in amorphous PE there is a transition between localized and delocalized states around the vacuum level providing an estimate of the mobility edge in amorphous PE. The localized states in our amorphous PE model extended down to $-0.33 \mathrm{eV}$ below the vacuum level.

This suggested that voids and amorphous regions trapped between crystalline phases could constitute very deep traps. For example, an electron at the bottom of the crystalline conduction band (at $0.65 \mathrm{eV}$ ) would see a state in a void (at the vacuum level, $\mathrm{V}=0.00 \mathrm{eV}$ ) as a trap of depth $-0.65 \mathrm{eV}$ and an amorphous region as containing traps as deep as $-(0.65$ $+0.33) \mathrm{eV}$. Of course if amorphous phases percolated the PE sample then the trap depth would be the difference between the ground state and the mobility edge, in previous work $\sim 0.33 \mathrm{eV}$, resulting in a finite mobility at room temperature.

Thus, the behaviour of PE will depend very sensitively on the local structure of the material and how such structures are distributed. Nonadiabatic simulations of an excess electron in amorphous PE at room temperature showed the spontaneous formation of localized small polaron states in which the electron was confined in a spherically shaped region with a typical dimension of $0.5 \mathrm{~nm}$. We computed the self-trapping energy to be $\sim-0.06 \mathrm{eV}$, with a lifetime on the time scale of a few 
tens of picoseconds. The smallness of the self-trapping energy suggested an adiabatic hopping mechanism assisted by phonons, as observed in the simulations. The contribution to the mobility due to hopping between these self-trapped states may well be of same order of magnitude as the mobility due to excited electrons above the mobility edge. ${ }^{11}$

The discussion above has illustrated the importance of electron transport through extended states in amorphous regions to an understanding of electron transport in PE as well as the role of conformational disorder in the trapping of electrons. In this paper we extend our study to include models of other structural phases of PE including lamella as well as the interfacial regions of PE between amorphous and lamella (rather than bulk orthorhombic crystal) regions and nanometre-sized voids. These interfacial and void structural motifs are thought to play an important role in dielectric breakdown. Note however that although nanometre sized holes (average radius, $\mathrm{r}_{\mathrm{h}}=0.343 \mathrm{~nm}$ ) are present, ${ }^{12}$ amounting to $13.2 \%$ of the total volume at $300 \mathrm{~K}$ in low density polyethylene (LDPE) from positron lifetime studies, ${ }^{13}$ the literature usually considers $\mu \mathrm{m}$ sized voids as initiators of mechanical and electrical breakdown. ${ }^{14}$ Through our simulations we identify charge-trapping regions in interfacial structures and look for correlations with local density. We wish to go beyond a simple characterisation of the trapping as Anderson localisation ${ }^{5}$ to identify, where possible, the microscopic features that cause the electron to localise in a particular region or regions. At present a clear connection between a localisation site and the local atomic structure only exists for inorganic materials. ${ }^{4}$ This microscopic structural understanding may permit the design of new synthesis and processing methods that can produce more resilient and reliable insulating materials. In what follows we refer to polyethylene phases (amorphous, lamella, etc.), these should be understood to represent the regions of metastable polyethylene morphologies found in the real material. The volume fraction of each phase present in real materials (high density polyethylene, low density polyethylene, and others) depends on the processing history.

The paper is organized as follows: Section II presents an overview of the methodologies we employ in these studies including sample preparation, some tests of the reliability of the model interactions, as well as details of our methods to compute and characterize the electronic states. Section III presents our results for how our calculated electronic properties (densities of states, mobility edges, electronic mobilities, etc.) depend on the different PE phase, and PE interface nano morphologies. We also present results that explore the correlation between electronic structure and local free volume in an attempt to learn how such properties might be engineered to enhance material properties. Our findings are discussed and conclusions presented in Sec. IV.

\section{METHODOLOGY}

\section{A. Polyethylene phases}

In this section we review the different types of nanoscale structures and interfaces that are relevant to modelling realis- tic, technologically important PE materials. We detail our approach to sample preparation for our simulation studies in the supplementary material. ${ }^{15}$ The model nano structures we consider include pure lamella, amorphous, and crystalline phases, as well as interfaces such as lamella-amorphous and "holes" or void spaces that are introduced into the pure phases.

A detailed account of the dominant morphologies of melt-crystallized polyethylene is given in Ref. 16. Inter-chain interactions lead to formation of stable folded lamellae structures. Large-scale mesoscopic spherulite structures form from packing of lamellae. The spherulite structure is the most prevalent morphology in the melt-crystallized polyethylene, which is the common method of producing PE films. The size of the spherulites generally depends on the details of the under-cooling process employed during crystallization. ${ }^{17}$ The diameter of the single spherulite varies considerably from sizes less than $10 \mu \mathrm{m}$ to nearly $100 \mu \mathrm{m}$ depending on a variety of factors such as heat treatment, nucleating agent, and molecular weight.

An individual spherulite is composed of radiating lamellae structures that are formed at the molecular level by folded chains. The interfacial regions between these lamellae structures typically involve layers of amorphous-like material located between the folded surfaces of the lamellae. In this paper we use simulation methods to explore the variation of single electron properties for different typical homogenous and heterogenous micro structures in PE with the goal of understanding how such structures influence the bulk insulating properties of these materials.

The PE simulations reported here assume that the structure of the material does not relax in the presence of the injected excess charge and that for the purposes of exploring the single electron states, the underlying heterogeneous structure of PE is critical. Thus, our simulations are performed using models of crystalline, lamella and amorphous PE structures their interfacial regions, and nanovoids. Representative structures employed in our simulations are presented in Fig. 1. As suggested above, any given real bulk sample may have amorphous, lamella, void and crystalline regions of various sizes depending on material processing details, molar mass differences, and a host of other conditions. ${ }^{16,18,19}$

\section{B. Simulation model}

All our MD simulations of the different phases of PE employed a united atom model force field developed by Siepmann et al. ${ }^{20}$ This force field has been used with success to predict the vapour-liquid equilibrium of long n-alkanes. Constant NPT (at 1 atm and $300 \mathrm{~K}$ ) simulations each of duration at least $10 \mathrm{~ns}$ and detailed in the supplementary material, ${ }^{15}$ gave stable structures for the different model phases. The computed densities from these simulations are reported in Table I and compare well with the known experimental densities for $\mathrm{PE}$, for example, the pure crystalline phase has density $1.01 \mathrm{~g} / \mathrm{cm}^{3}$, Ref. 21 , and high density PE is reported in the range $0.94-0.97 \mathrm{~g} / \mathrm{cm}^{3}$.

Our procedure to generate representative amorphous PE samples is detailed in the supplementary material ${ }^{15}$ and 


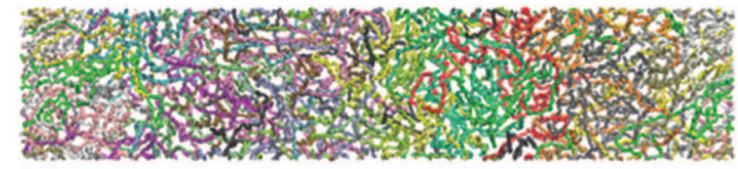

(a)

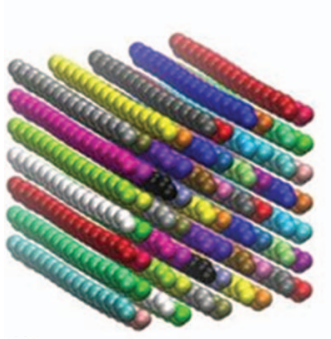

(b)

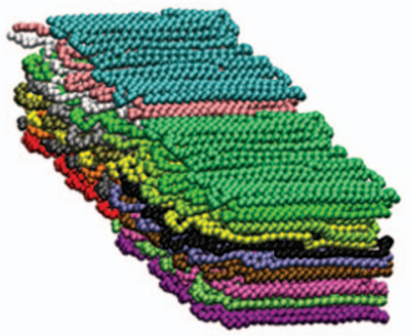

(c)
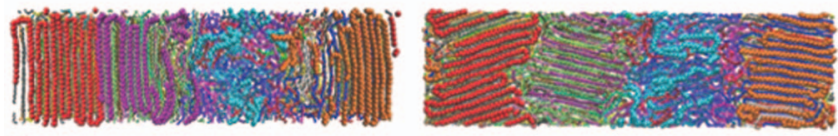

(d)

(e)

FIG. 1. This figure shows simulations cells different phases of polyethylene. (a) amorphous phase; (b) crystalline phase; (c) lamellae phase; (d) lamellaeamorphous (interfacial parallel); (e) lamellae-amorphous (interfacial perpendicular). Individual polymer chains are each coloured differently for clarity.

TABLE I. Densities of simulated phases.

\begin{tabular}{lc}
\hline \hline Phase $(300 \mathrm{~K})$ & Density $\left(\mathrm{g} / \mathrm{cm}^{-3}\right)$ \\
\hline Crystalline & 1.028 \\
Lamellae & 0.958 \\
Interfacial-perpendicular & 0.934 \\
Interfacial parallel & 0.922 \\
Amorphous & 0.860 \\
\hline \hline
\end{tabular}

involved ramping the temperature of an initially prepared lamellae sample up and down between $300 \mathrm{~K}$ and $500 \mathrm{~K}$ at constant pressure over $20 \mathrm{~ns}$. This lamella structure is in agreement with the morphology recently observed using torsional-tapping-atomic-force microscopy. ${ }^{22}$ A broad melting curve was observed centred around $390 \mathrm{~K}$, in reasonable agreement with the experimental reported melting temperature $(398 \mathrm{~K})$ observed in lamellae phases of blends of branched and linear PE. ${ }^{14}$ Super-cooling from this melt at constant pressure gave our representative amorphous samples (see Fig. 2). The supplementary material ${ }^{15}$ also reports various structural properties of the different phases and demonstrates good agreement between our computed static structure factor and experimental results.

Polyethylene contains a significant fraction $(13.2 \%$ by volume, $300 \mathrm{~K}$, LDPE) of nanometre sized holes. ${ }^{12}$ In order to explore their influence on the electron density of states in PE we have produced nanometre sized voids in our simulated amorphous phase by expanding a test particle. The details of these calculations are presented in the supplementary material. ${ }^{15}$ In addition to providing representative configurations for our studies of the effects of holes on the density of states in PE, thermodynamic integration of calculated free energies yields an estimate of the surface tension of amorphous $\mathrm{PE}$ of $36 \mathrm{mN} / \mathrm{M}$ that agrees well with experimental results $35.7 \mathrm{mN} / \mathrm{M}$ for unbranched $\mathrm{PE}^{23}$ at $20{ }^{\circ} \mathrm{C}$ giving us further confidence that our simulation model gives reliable predictions for the bulk properties and structure of phases of PE.

\section{Calculation and characterisation of electronic states}

We have used a block Lanczos diagonalisation algorithm $^{24}$ to compute the excess electronic states of

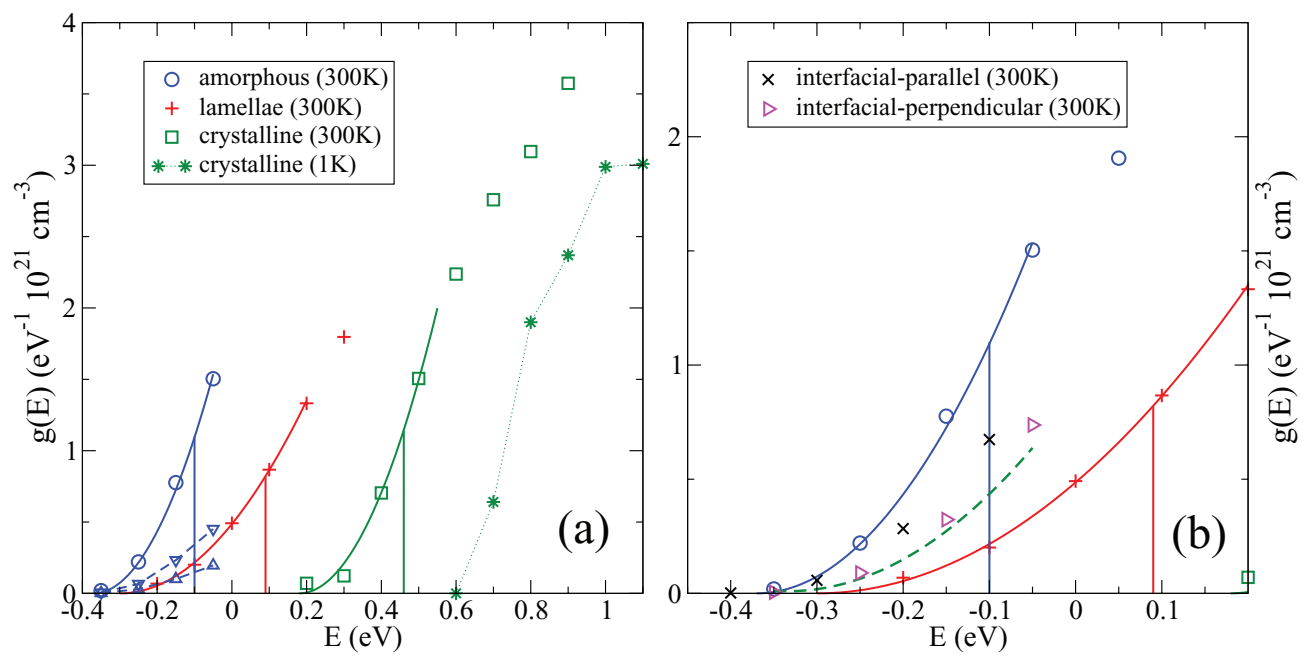

FIG. 2. Computed densities of states for various PE structural motifs: (a) amorphous (circles), lamellae (pluses), and crystalline (squares and stars) samples; (b) amorphous, lamellar, and interfacial samples. The crosses and the triangles depict to the density of states of lamellae-amorphous perpendicular and the lamellae-amorphous parallel interfacial samples, respectively. The solid lines show the pure phase quadratic fits detailed in the text. The dashed line in (b) shows the DoS obtained by weighting the lamella and amorphous DoS by their volume fraction in the interfacial phase (75\%, 25\%). The dashed lines (with triangles) in (a) show the lamellar DoS obtained by weighting the crystalline-amorphous DoS by the estimated volume fractions (87\%, 13\%) (triangles up) and $(70 \%, 30 \%)$ (triangles down), see the text. All densities of states are computed as averages over 15 independent configurations of the phase, except for the interfacial states where 20 independent configurations were used. We estimate the statistical error as $\pm 0.05 \mathrm{eV}$. The zero of energy corresponds to the vacuum level. ${ }^{11}$ 
the static configurations of different model PE material motifs generated using the molecular dynamics simulations outlined above. This approach calculates the excess electronic state wave functions and energies using a semi empirical pseudo potential model to describe the excess electronmolecule interactions. ${ }^{11}$ We assume that the concentration of excess electrons is very low, so that correlations between them can be safely ignored. Details of these calculations are outlined in the supplementary material. ${ }^{15}$ The excess electronic state energies computed for ensembles of representative configurations of the different PE phases are accumulated in histograms giving densities of states (DoS) per unit energy, in each spin direction, per unit volume, $g(E)$, for the different phases. These DoS results can be employed to compute various excess electronic properties such as the mobility, $\mu$, in the different phases using, e.g., the following result obtained from the Kubo-Greenwood formula:

$$
\mu=\frac{\pi e \hbar^{3} \Omega \beta}{m^{2}} \frac{\int K^{2}(\Omega, E) g^{2}(E) e^{-\beta E} d E}{\int g(E) e^{-\beta E} d E} .
$$

Here, $\Omega$ is the system volume, $\beta=1 / k_{B} T$, and $\hbar^{2} K^{2}$ $=\left|\left(p_{x}\right)_{i j}\right|_{a v}^{2}=\frac{1}{N_{\Delta}^{2}} \sum_{i, j}\left|\left\langle\psi_{i}\left|p_{x}\right| \psi_{j}\right\rangle\right|^{2}$ is the mean square momentum matrix element between pairs of different states with similar energy, averaged over all states in the energy interval $\Delta$ around $E$. These momentum matrix elements provide our criterion ${ }^{11}$ for determining above what energy (the so-called mobility edge, $E_{c}$ ) the excess electron states become extended and begin to make non-vanishing contributions to the mobility. Below the mobility edge the excess electronic states are localized and the momentum matrix elements connecting such localized states vanish so $K^{2}=0$. Extended states with energies $E>E_{c}$, on the other hand, generally have mean square momenta that are at inversely proportional to the volume, i.e., $K^{2} \propto 1 / \Omega$, resulting in non-zero mobility values that are independent of volume according to Eq. (1). The criterion we use to identify extended states ${ }^{11}$ makes use of the realization that for such states the surface flux term

$$
\Phi_{x}^{i j}\left(x_{B}\right)=-\frac{i \hbar}{2} \int_{x=x_{B}} d y d z\left(\psi_{i}^{*} \frac{\partial \psi_{j}}{\partial x}-\psi_{j} \frac{\partial \psi_{i}^{*}}{\partial x}\right)
$$

dominates the magnitude of the momentum matrix element $\left\langle\psi_{i}\left|p_{x}\right| \psi_{j}\right\rangle$ compared to the volume contribution. For localized states $\Phi_{x}^{i j}\left(x_{B}\right)$ is small compared to the momentum matrix element and it is strongly dependent on the position of the boundary, $x_{B}$. Once we reach states with sufficiently high energy so that $\Phi_{x}^{i j}\left(x_{B}\right)$ dominates the magnitude of the momentum matrix elements and it becomes independent of $x_{B}$ we have identified states with energies at or above the mobility edge, $E_{c}$. Details of the general implementation of this approach can be found in Ref. 11.

As will be demonstrated in the results section and observed in our earlier work, ${ }^{11}$ for the single electron PE systems considered here, the typical range of state energies are a few tenths of an electron volt and at room temperature $k_{B} T$ $=0.026 \mathrm{eV}$. Under these circumstances the exponentials in the integrals in the numerator and denominator of Eq. (1) decay very rapidly. Consequently, the integral in the numerator is dominated by the small range of energies around $E_{c}$, i.e., $E_{c}$ $\leq E \leq E_{c}+\Delta$ (where $K^{2}$ first becomes non-zero), while the denominator integral is dominated by a similar small range of energies around $E_{0}$ (where $g(E)$ first becomes non-zero). With these approximations it is easy to show ${ }^{11}$ that the mobility has the form

$$
\mu=\mu_{0} \exp \left[-\beta\left(E_{c}-E_{0}\right)\right],
$$

where the activation energy, $E_{A}=E_{c}-E_{0}$, is just the gap between the mobility edge and the ground state energy. Evaluating the approximate integrals in the numerator and denominator and taking the limit as $\Delta \rightarrow 0$ we obtain the following explicit form for the pre-factor within the approximations outlined above,

$$
\mu_{0}=\frac{\pi e \hbar^{3} \Omega \beta^{3}}{2 m^{2}} K^{2}\left(\Omega, E_{c}\right) g\left(E_{c}\right)\left(E_{c}-E_{0}\right)^{2},
$$

i.e., the pre-factor depends on the DoS at the ground-state energy (assumed to vary quadratically as detailed below), its value at the mobility edge, and the average electron momentum at the mobility edge. ${ }^{11}$

\section{RESULTS}

\section{A. Characteristic DoS for different structural motifs}

Single electron DoS for various pure phases of PE are presented in Fig. 2. Starting with the crystalline phase at $T=1 \mathrm{~K}$ on the right hand, high energy, side of Fig. 2(a), the DoS generally shift progressively to lower energy as the PE density decreases and/or disorder increases. For the low temperature perfect crystal all states are extended and the energy of the lowest state, $E_{0}$, and the energy of the lowest extended, or conducting state, $E_{c}$ (the mobility edge) coincide and $E_{0}$ $=E_{c}=+0.6 \mathrm{eV}$ corresponding to the bottom of the conduction band, consistent with our earlier calculations. ${ }^{11}$ Increasing the temperature to $T=300 \mathrm{~K}$ shifts the crystalline phase DoS down in energy so that the lowest energy state now has $E_{0} \approx+0.18 \mathrm{eV}$ and creates a set of localised states below the mobility edge at $E_{c}=+0.46 \mathrm{eV}$ as indicated by the vertical line separating localized and delocalized states as determined by our criterion based on Eq. (2). This value is in excellent agreement with the value of $+0.5 \pm 0.1 \mathrm{eV}$ usually quoted for the bottom of the conduction band in low density PE from low-energy electron transmission and secondaryelectron emission experiments on long chain alkanes at room temperature. ${ }^{25}$ Note however that we have shown that the conduction states are different in crystalline PE and long chain alkanes as in crystalline PE the conduction state is inter-chain while in the long chain alkane it is interlayer, ${ }^{11}$ so it is not clear that the energy of the bottom of the conduction band in crystalline (or lamella) PE is known from experiment. The low energy initial rise of the DoS for the crystalline phase at room temperature can be fitted to the quadratic form $g_{\text {crys }}(E)$ $=14.6\left(E-E_{0}\right)^{2} \mathrm{eV}^{-1} \times 10^{21} \mathrm{~cm}^{-3}$, with $E_{0}=+0.18 \mathrm{eV}$, and the difference in energy between states at the mobility edge and the localised ground state energy, the activation energy for transport, is $E_{A}^{c r y s}(T=300 \mathrm{~K})=\left(E_{c}-E_{0}\right) \approx+0.28 \mathrm{eV}$. This is somewhat larger than the $\approx 0.1 \mathrm{eV}$ found for electrons trapped at $300 \mathrm{~K}$ in trans-gauche crystal defects by Serra 
et al. ${ }^{26}$ using Car-Parrinello techniques, however the present system is much larger (2100 $\mathrm{CH}_{2}$ units compared to 56) so it can in principle accommodate a wider range of thermal disorder. Further, these Car-Parrinello calculations allow the crystalline PE configuration to relax in the presence of the excess electron where as our studies ignore this relaxation. A contribution to this difference could also come from the approximate BLYP functional employed in the Car-Parrinello calculations which we expect to be less reliable than the semiempirical pseudo potential we use in our calculations which is fitted to reproduce the density dependence of the ground state energy in a variety of liquid alkanes.

The introduction of folded chains in the lamellae phase at $\mathrm{T}=300 \mathrm{~K}$ causes an even more significant shift of the DoS down in energy by a further $\approx 0.5 \mathrm{eV}$ relative to the $300 \mathrm{~K}$ crystal phase, making the lamella ground state $E_{0}=-0.3 \mathrm{eV}$ at this temperature, with the mobility edge at $E_{c}=+0.09 \mathrm{eV}$. So the activation energy for transport is $E_{A}^{\operatorname{lam}}(T=300 K)$ $=\left(E_{c}-E_{0}\right) \approx+0.39 \mathrm{eV}$ and the low energy tail of the DoS can be fitted by a significantly more shallow rising quadratic form, $g_{\text {lam }}(E)=5.4\left(E-E_{0}\right)^{2} \mathrm{eV}^{-1} \times 10^{21} \mathrm{~cm}^{-3}$. Lowering the PE density to that of the pure amorphous phase at $\mathrm{T}=300 \mathrm{~K}$ further shifts the DoS to lower energy so that the ground state is now at $E_{0}=-0.37 \mathrm{eV}$, and the low energy tail of the DoS has the form $g_{a m}(E)=15\left(E-E_{0}\right)^{2} \mathrm{eV}^{-1}$ $\times 10^{21} \mathrm{~cm}^{-3}$, with the mobility edge at $E_{c}=-0.1 \mathrm{eV}$, making the activation energy for transport in the room temperature amorphous phase $E_{A}^{a m}(T=300 K)=\left(E_{c}-E_{0}\right)$ $\approx+0.27 \mathrm{eV}$. These quadratic fits of the DoS are used in the mobility calculations reported in Sec. III B, and as such only cover the energy interval from the ground-state to the mobility edge.

The results presented in Fig. 2 suggest a rule of thumb that apparently applies quite generally for the excess electron states in these different PE phases. Once the DoS reaches a critical value above about $1 \mathrm{eV}^{-1} \times 10^{21} \mathrm{~cm}^{-3}$ we see the onset of extended states as signaled by our delocalization criterion in Eq. (2) and the establishment of a mobility edge. This observation is consistent with the significantly larger activation energy, $E_{A}$, for excess electron transport in the lamellar phase of PE compared to that for the crystal or amorphous phases reported above. This occurs because the curvature of the initial quadratic rise of the DoS is about three times greater for the crystal and amorphous phases compared to the much slower rise in the DoS for the lamellar phase as is clearly apparent in Fig. 2. As a result, in the lamellar phase, we do not reach the extended states till an activation energy of $E_{A}^{\text {lam }}=+0.39 \mathrm{eV}$, i.e., more than $40 \%$ larger than the typical activation energies found in the crystal and amorphous phases $\left(E_{A}^{\text {crys } / a m} \approx 0.27-0.28 \mathrm{eV}\right)$. As we will see in Sec. III B, these differences in the variation of the DoS with energy have a profound influence on the excess electron mobility in the different phases of PE.

It might be thought that the interfacial DoS would be intermediate between the lamellar and amorphous phases, with the ground state close to that of the pure amorphous phase (where it has its lowest value). Instead, however, we find that the lowest energies are slightly lower than those found the amorphous phase. On the other hand, the DoS at higher energies follows approximately that obtained by weighting the lamellar and amorphous DoS by their volume fraction in the interfacial phase $(75 \%, 25 \%)$, especially the interfacialperpendicular setup, see the dashed line in Fig. 2(b). A larger fraction of amorphous phase is needed, however, for a better fit in the interfacial-parallel case, which is not unreasonable given the amount of lamellar distortion shown in Fig. 1(d).

To summarize, in this section we have found that the overall position of the DoS in energy for PE is predominately influenced by the atomic density with the ground state energy decreasing as the solvent density decreases (see Table I). The shape of the DoS, however, shows a significant dependence on the nature of the local structure of the particular phase of PE. In Sec. III B we will explore how the variation in the shape of the DoS has a profound influence on the excess electron mobility of the different PE phases so that the mobility is not at all a simple function of PE density.

\section{B. Dependence of excess electron mobility on local PE structure}

The electrical conductivity of each phase can be calculated from the DoS using the Kubo-Greenwood formula as outlined in Sec. II. ${ }^{11}$ For our single electron PE systems at room temperature, the activation energies for transport in the various phases reported above, and summarized in Table II below, are all significantly larger than the thermal energy $\left(k_{B} T\right.$ $=0.02585 \mathrm{eV})$, i.e., $E_{A}=\left(E_{c}-E_{0}\right) \gg k_{B} T$. Under these circumstances, as outlined in Sec. II, ${ }^{11}$ the mobility can be computed using the simplified expressions in Eqs. (3) and (4).

In Table II we list the ground state, mobility edge, and activation energies as well as the mobilities of each phase, computed using the quadratic fits reported in Sec. III A. Note that the pseudo-potential energy resolution produces an uncertainty in the mobility of about one order of magnitude, due to the exponential dependence of the mobility on the activation energy. Nevertheless, we expect these values to be meaningful for comparison purposes between phases. Remarkably, the excess electron mobilities in the crystalline and amorphous phases at room temperature are very similar. However, in the lamellar phase, despite displaying the largest pre-exponential factor, $\mu_{0}$, due to the significantly larger activation energy required to take an electron from the ground-state to states at the mobility edge in the lamellar phase, the computed electron mobility in this phase is some 40 times smaller than the electron mobilities calculated for the room temperature amorphous or crystalline phases.

The results presented in Figures 3-5 give a microscopic structural understanding of the origin of this significantly

TABLE II. Electron ground state, $\left(E_{0}\right)$, mobility edge, $\left(E_{c}\right)$, and activation energies, $\left(E_{A}\right)$, mobility pre-exponential factors, $\left(\mu_{0}\right)$, and mobilities, $(\mu)$, at room temperature $\left(k_{B} T=0.02585 \mathrm{eV}\right)$.

\begin{tabular}{lccccc}
\hline Phase $(300 \mathrm{~K})$ & $E_{0}(\mathrm{eV})$ & $E_{c}(\mathrm{eV})$ & $E_{A}(\mathrm{eV})$ & $\mu_{0}\left(\mathrm{~cm}^{2} / \mathrm{V} \mathrm{s}\right)$ & $\mu\left(\mathrm{cm}^{2} / \mathrm{V} \mathrm{s}\right)$ \\
\hline Crystal & 0.18 & 0.46 & 0.28 & 394 & 0.0080 \\
Lamella & -0.3 & 0.09 & 0.39 & 534 & 0.0002 \\
Amorphous & -0.37 & -0.1 & 0.27 & 223 & 0.0070 \\
\hline \hline
\end{tabular}




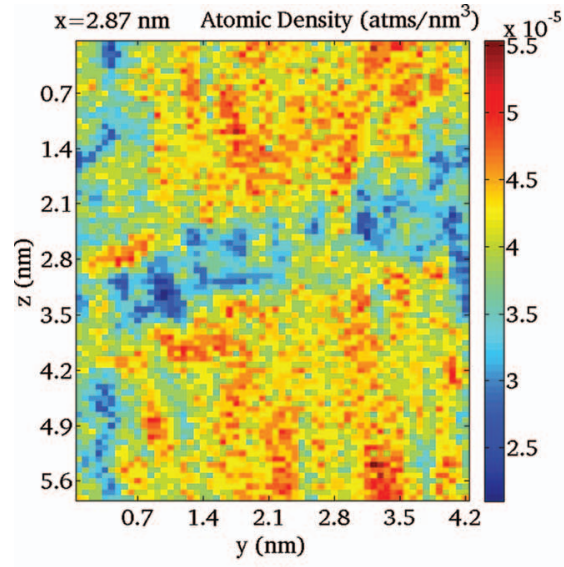

(a)

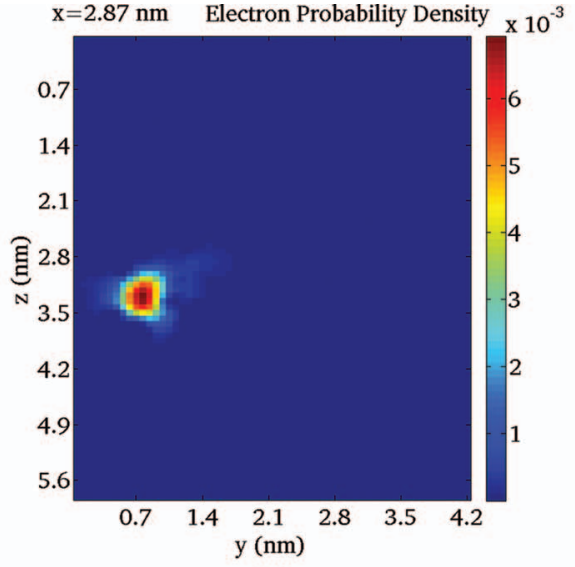

(b)

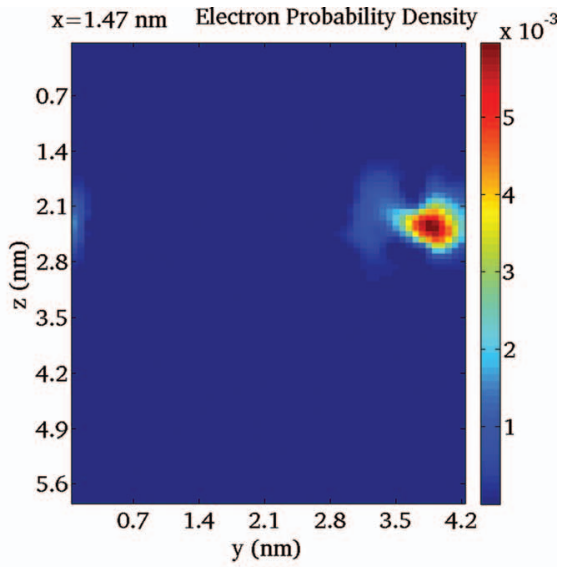

(c)

FIG. 3. (a) Colour contour plot of local atomic density in a slab from a lamellar phase simulation box that contains the lowest few energy states. Panels (b) and (c) show color contour plots of the electron density for the ground and excited state, respectively, in this lamellar phase slab. These low lying trap states form at the simulated interface between adjacent lamella.

reduced mobility of the lamellar phase compared to that in the crystal or amorphous phases. In our simulation model of a typical configuration of the lamellar phase (Fig. 3(a)) we see an extended region of significantly reduced atomic density between the higher density lamellar layers. From the electron density contour plots in Figs. 3(b) and 3(c) it is clear that the lowest energy states are trapped in this inter-lamellar region that runs transverse to the orientation of the molecules in the lamellar regions. For this configuration the inter-lamellar region lies in the $x-y$ plane and is $1-1.5 \mathrm{~nm}$ in thickness in the $\mathrm{z}$-direction, stretching from around $\mathrm{z}=2.0$ to $\mathrm{z}=3.5 \mathrm{~nm}$ in these figures. Figure 4 presents results for the same lamellar configuration as in Fig. 3 except that it focuses on the lowest energy state in this configuration that is delocalized over the length scale of the lamellar simulation as determined by the criterion associated with Eq. (2). In Fig. 4(c) we see that the first extended state at the mobility edge is elongated along the x-direction. Figures 4(a) and 4(b) present color contour maps of the atomic density and the excess electronic pseudo-potential projected onto the slab containing this first extended electronic state. The striations of high atomic density and electron-molecule repulsion associated with the lay- ers of polymer and the ragged channel formed at the interface along which this state extends are evident.

According to the discussion of Sec. III A the mobility is determined primarily by thermal excitation from the low energy states localized in the inter-lamellar region to the extended states at the mobility edge that are apparently also confined to the inter-lamellar channel. For our estimates of mobility to be reliable we assume that such typical channellike inter-lamellar structures that support these lowest energy extended states are macroscopic in size, i.e., these channel structures percolate along the length of the macroscopic interface and electrons that enter such inter-lamellar structures with sufficient energy (above the mobility edge) will undergo macroscopic transport here.

Comparing the atomic density contour plots in the lamellar regions of Figs. 3(a) or 4(a) with those for the crystalline phase displayed, for example, in Figs. 5(a) and 6(a) we see that the dense lamellar regions show strong local ordering, similar to the layered oriented polymer chain structure observed in the room temperature crystalline phase. Consequently, considering the DoS plots in Fig. 2(a), there is a significant energy barrier of almost $0.75 \mathrm{eV}$ to take electrons

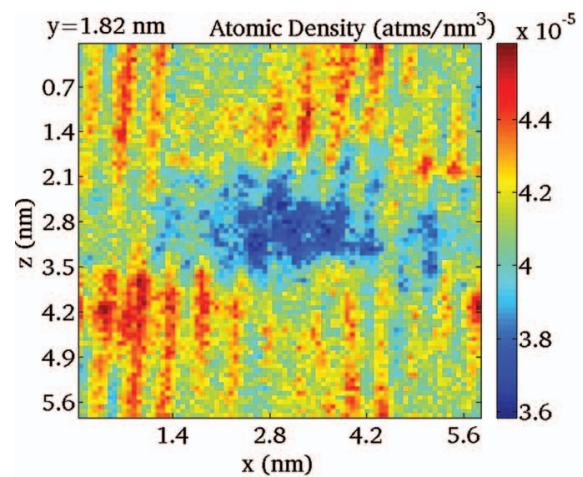

(a)

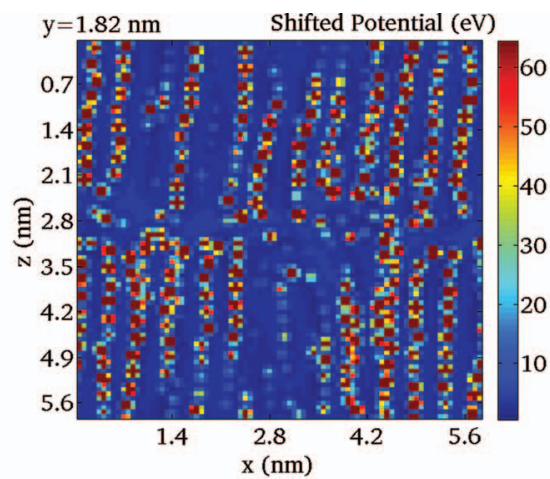

(b)

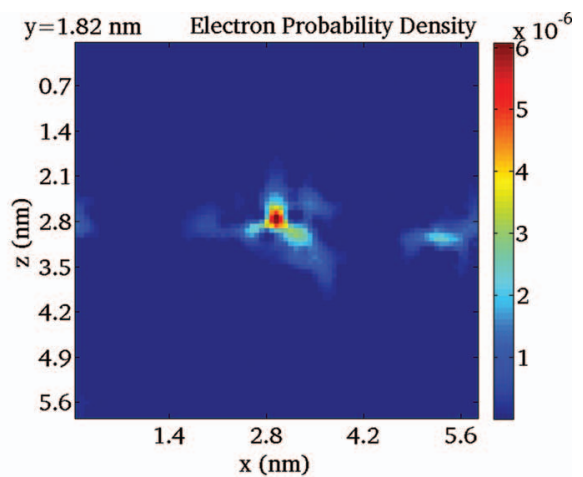

(c)

FIG. 4. Same lamellar configuration and information as in Fig. 3 but now showing details of the slab in which the first extended state (state 11) is observed. These extended states also form at the simulated interface between adjacent lamella. 


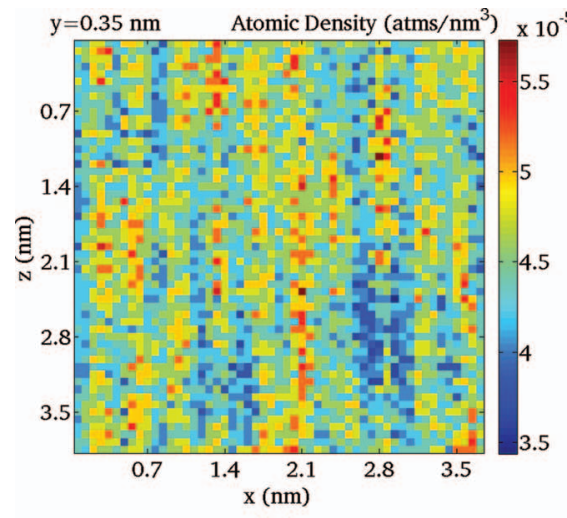

(a)

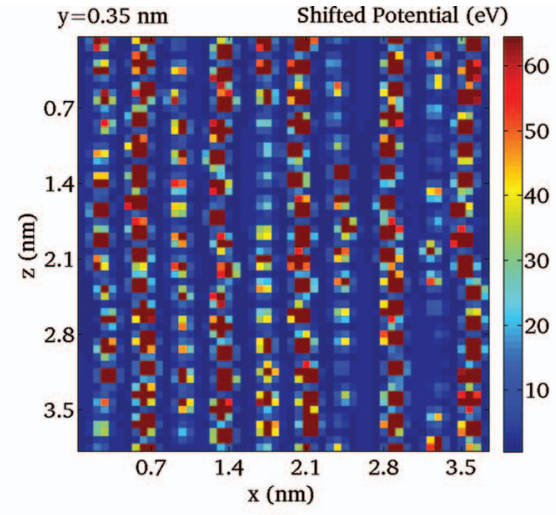

(b)

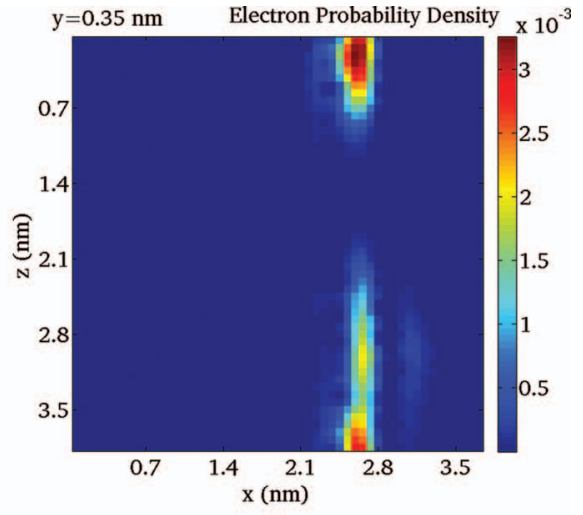

(c)

FIG. 5. (a) Color contour plot showing local atomic density in a slab from a room temperature crystal phase simulation box that contains the lowest energy state. (b) Pseudo potential energy contour plot for this slab. (c) Contour plot of ground state electron density in this room temperature crystal phase slab.

from the lowest energy states supported by the inter-lamellar region (at around $-0.3 \mathrm{eV}$ ), and inject them into the conducting states of the crystal-like lamellar regions that are typically near energies of about $+0.45 \mathrm{eV}$ (assuming that the lamella have similar electronic properties to the crystal). The activated mobility of the lamellar structured material thus arises from exciting localized states that are trapped within the interlamellar region, to extended states, e.g., Fig. 4(c), that are also confined to these inter-lamellar regions. Thus, our calculations suggest that excess electrons in lamellar structured PE at typical thermal energies are confined to states that occupy the inter-lamellar regions. If we approximate the inter-lamellar region by the same volume of the amorphous phase and the intra-lamellar region by the room temperature crystal, we can predict the lamellar DoS as shown in Figure 2(a). As the crystal DoS does not start until energies well above the mobility edge of the lamella phase, the lamella DoS is given only by the amorphous DoS multiplied by the amorphous volume fraction in the inter-lamella phase. The triangles in Fig. 2(a) show the predictions with two different estimations. Before equilibration, the length of the intra-lamellar region is easily measured from the length of the all-trans chains as $5.01 \mathrm{~nm}$. Dividing this length by the size of the NPT-equilibrated simulation box in the direction of the chains leads to an estimation of the crystalline-amorphous volume fraction of $(87 \%, 13 \%)$, which is shown with triangles-up in Fig. 2(a). Like in the interfacial DoS prediction, this method underestimates the real amount of disorder present in the configuration, a fact that is also confirmed by simple inspection of Figs. 3(a) and 4(a). A simple approximate estimation of $(70 \%, 30 \%)$ for the crystallineamorphous volume fraction based on these representations produces the triangle-down data shown in Fig. 2(a).

Figures 5 and 6 present the wave function, local atomic density and excess electron pseudo potential for the ground and first extended state of a typical room temperature crystalline PE configuration. The energy required to promote electrons from the localized low energy states in the crystal, where thermal disorder along the chains give rise to potential energy barriers that "pinch off" the low energy localized wave functions (see, e.g., Fig. 5(c)), to extended, conducting states of the room temperature crystalline configurations (e.g., Fig. 6(c)) is typically $E_{A} \approx 0.3 \mathrm{eV}$ in this higher density manifold of states. This is to be compared with excitation between localized and extended states in the confined inter-lamellar region with its more sparse manifold of low energy states where $E_{A} \approx 0.4 \mathrm{eV}$. These structural differences thus result

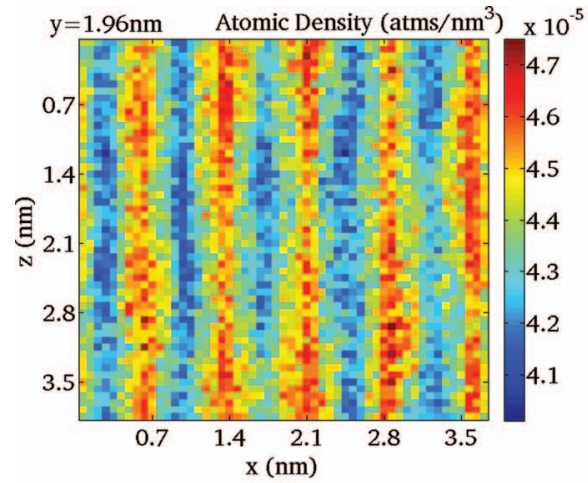

(a)

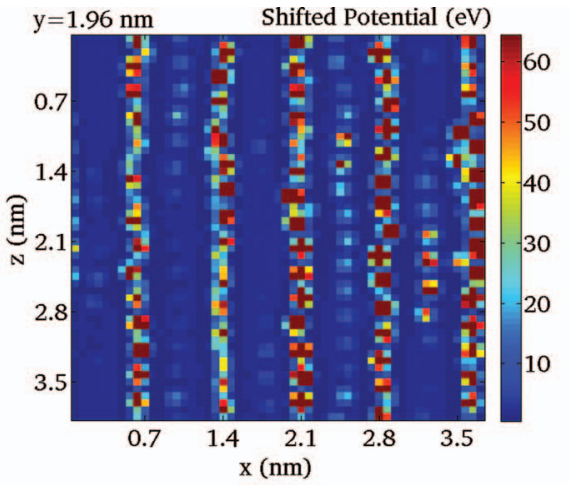

(b)

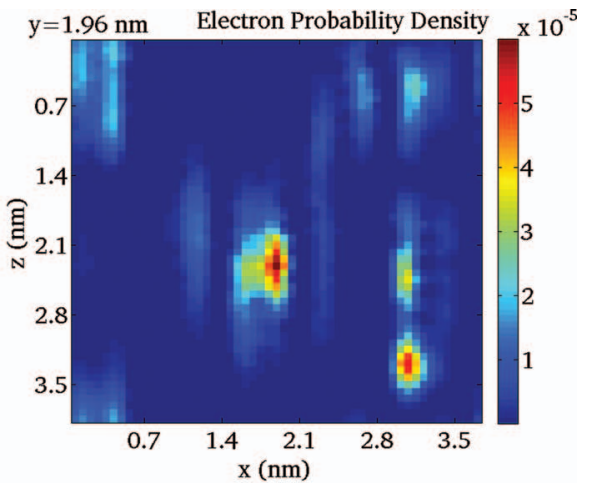

(c)

FIG. 6. Same quantities as in Fig. 5 computed for the same configuration except here results are displayed for the first extended state for this configuration as determined by criterion associated with Eq. (2). 


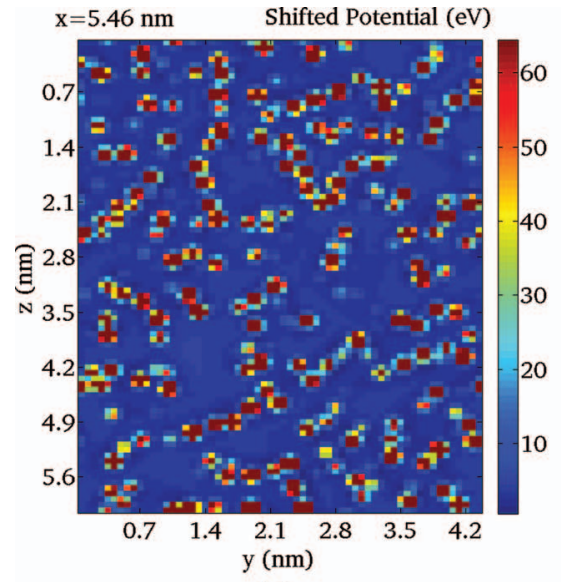

(a)

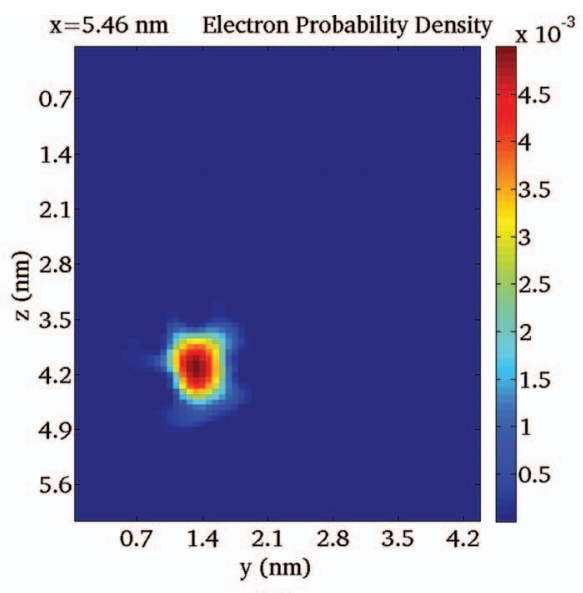

(b)

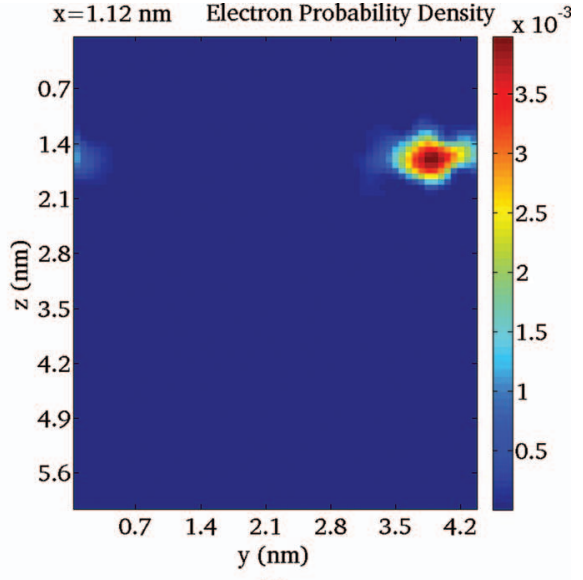

(c)

FIG. 7. (a) Colour contour plot showing local pseudo potential energy in a slab from a room temperature amorphous phase simulation box that contains the lowest few energy states. Panels (b) and (c) show the contour plot of the ground and first excited state electron density in this amorphous phase slab.

in the considerable difference in electron mobility we have observed between the heterogeneous lamellar phase and the homogenous phases.

Interestingly, in the amorphous, lowest density phase (see Fig. 7) we see a similar pattern of states to that of the room temperature crystal involving low energy states localized in different regions, transitioning to higher energy extended states (see Fig. 8) in which the electron density percolates throughout the porous-like three dimensional homogenous amorphous structure. The activation energy for transport in the crystal and amorphous phases is thus similar, while the nanostructured heterogeneity at the inter-lamellar boundaries in the lamellar phase gives a more dilute manifold of states in this confined region that leads to the larger activation energies responsible for the dramatic reduction in excess electron mobility in the lamellar phases compared to the homogenous crystalline or amorphous phases.

\section{Localization and local properties of polyethylene}

We now turn to the relationship between the local properties of polyethylene and the localization region of the electron. Currently there is no way of predicting where an electron will localize except by solving the Schrodinger equation. However several authors have noted an association with regions of lower atomic density and our simulations show that, for example, ground state electrons localize preferentially in regions of polyethylene with a local density (calculated at each grid point in a cubic volume of side $0.5 \mathrm{~nm}$ centred on the excess electron grid points as the number of atoms with centres in the cube) $\sim 25 \%$ lower than the mean density for the noncrystalline phases. Localisation lengths vary but are $\sim 0.5 \mathrm{~nm}$ in the low energy regions and we know from positron annihilation experiments that a significant fraction of the free volume of polyethylene is made up of cavities with radii smaller than $0.5 \mathrm{~nm}$

We have measured the free volume distribution in the homogeneous phases by identifying first local cavities in the material, and then assigning them a cavity radius. Using the excess-electron grid lattice, we located cavities by looking for grid points at local minima of the atomic density. The cavity radius was computed by averaging over the distance from the grid point to the carbons, which are closer than the position given by the first peak of the radial distribution function. Due to the cavity radius definition and PE microscopic structure (the carbon-carbon bond distance is $0.15 \mathrm{~nm}$ ), the

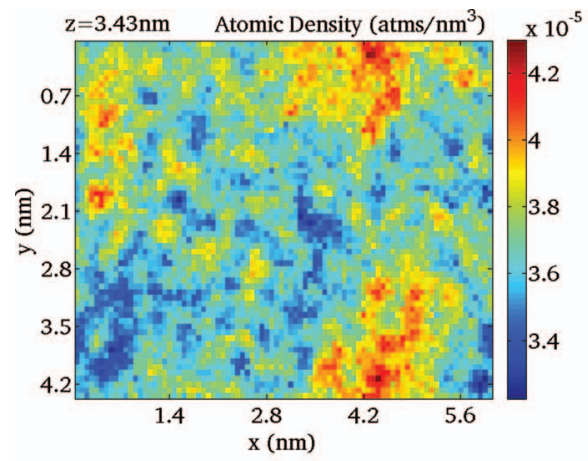

(a)

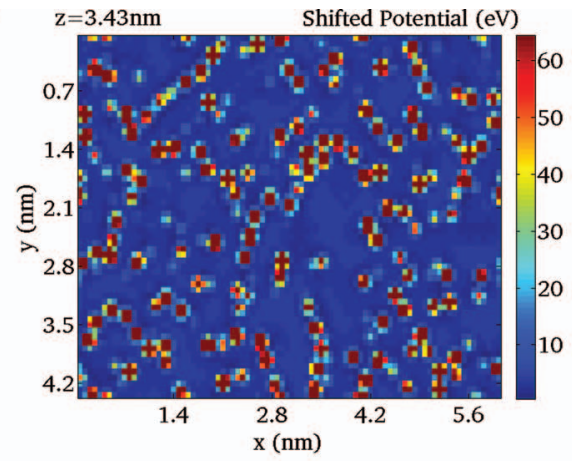

(b)

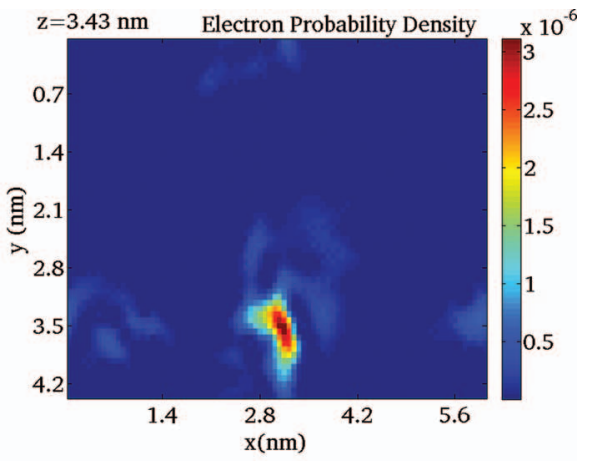

(c)

FIG. 8. Same amorphous configuration as in Fig. 7 only now focusing on a region around the first extended excited state. Colour contour plots showing (a) local atomic density, (b) local pseudo potential energy, and (c) probability density of the first extended state in this amorphous phase slab. 


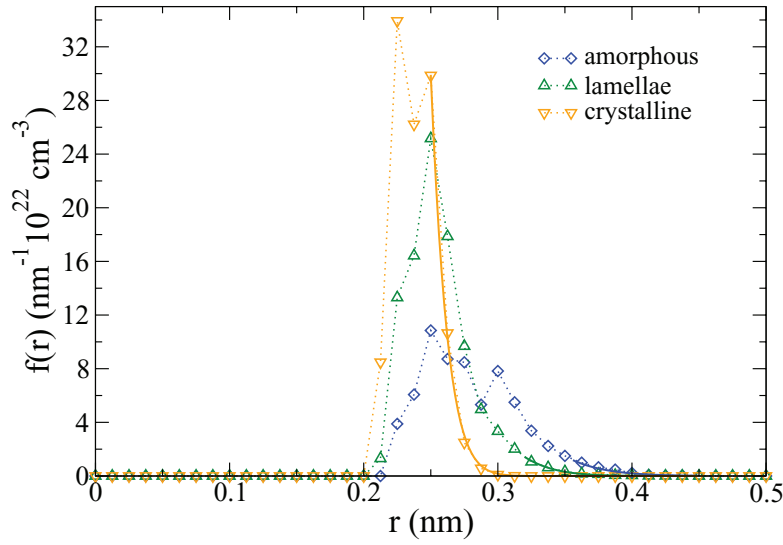

FIG. 9. Number cavity density $f(r)$ as a function of the average cavity radius for amorphous (diamonds), lamellae (triangles up), and crystalline (triangle down) at room temperature. The solid lines indicate the Gaussian fits that are used for the data shown in Fig. 12. Note these distributions are similar to but not directly comparable with those estimated from positron lifetime spectroscopy. ${ }^{13}$

smallest cavity possible involves two carbons, resulting in an average cavity radius of about $0.2 \mathrm{~nm}$, the minimum radius detected with this method.

Figure 9 shows the calculated number of cavities per unit of volume and unit of radius in amorphous, lamellar, and crystalline samples at room temperature. The data have been averaged over 15 configurations in each phase. A larger number of cavities of larger radius are found in the phases with the greater disorder, as expected.

Since the lowest energy levels of the excess electron are usually found in regions displaying low values of the local atomic density, it is tempting to associate each localized state with a cavity. Figure 10 shows the resulting localised energy function for each phase. Cavities were assigned by finding the nearest to the center of mass of the localized state. It can be seen in the figure that the data for the unperturbed phases matches naturally with the energies associated with artificial

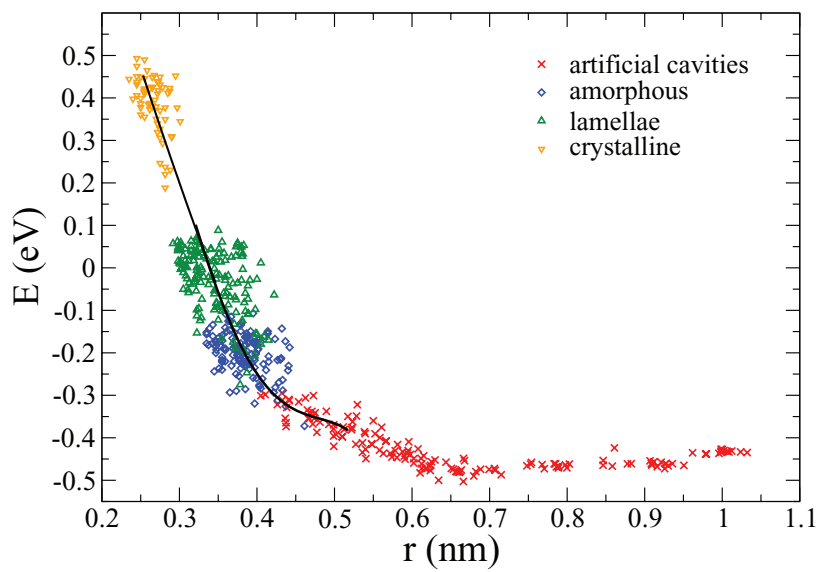

FIG. 10. Energies of the localized states as a function of the average cavity radius in amorphous (diamond), lamellae (triangle up), and crystalline (triangle down) phases at room temperature. The solid line is a linear fit to a polynomial of degree 4 , being piecewise defined through the three bulk phases. The crosses correspond to the ground-state energies of the electronic states sitting at artificial cavities. cavities. These large artificial and spherical cavities were generated in the simulations by introducing an extra particle with a Gaussian repulsive interaction with the PE carbons which is removed from the simulation once the cavity has formed.

Given the energy as a function of the cavity radius, the next step is to calculate the electronic density of states from the measured cavity density $f(r)$. Associating a localized state of energy $E$ with a single cavity of radius $r$ leads to a prediction of the DoS given by $g(E)=f(r) \mathrm{dr} / d E$. However, assuming a 1:1 relationship between a cavity and an electronic localized state yields a clear overestimation of the density of states: From a factor of 2 or 3 in the amorphous and lamella phases, to an order of magnitude larger in the crystalline phase. In other words, there are fewer states than cavities. The reason for this can be easily understood by looking at Fig. 11, where the localization length, $L=\left(\left\langle r^{2}\right\rangle-\langle r\rangle^{2}\right)^{\frac{1}{2}}$, is plotted as a function of the cavity radius. This length is about a quarter of the real wave-function extent in space. To see this, let us assume that the wave function has the same form as the ground-state of an electron in a spherical infinite square well of radius $r_{L}$. Then, the localization length would be $L=0.53 r_{L}$. We consider $r_{L}=2 L$ as a good estimation of the electronic radius in our simulations. Indeed, the probability that the electron is inside a sphere, centered at the centre of mass of the localized state, with radius $r=L$ is usually about $70 \%$ in our simulations, whereas most of the remaining probability (up to a total of about $96 \%$ ) is in the region between $r=L$ and $r=2 L$.

In all cases, the electronic radius $r_{L}=2 L$ is much larger than the cavity radius $r$. Rather than just penetrating inside the cavity surface, each electronic state is usually sitting at more than one cavity, invalidating the assumed 1:1 correspondence between cavities and localized states. However, for a given localization length the associated cavities were of similar sizes.

We can use this observation to provide a correction to the predicted density of states from the cavity distribution. For each localized state of radius $r_{L}$ centered at a cavity of radius $r_{0}$, there will be a number of extra cavities in the region $r_{0}<r<r_{L}$. Let us denote as $N$ the number of those extra

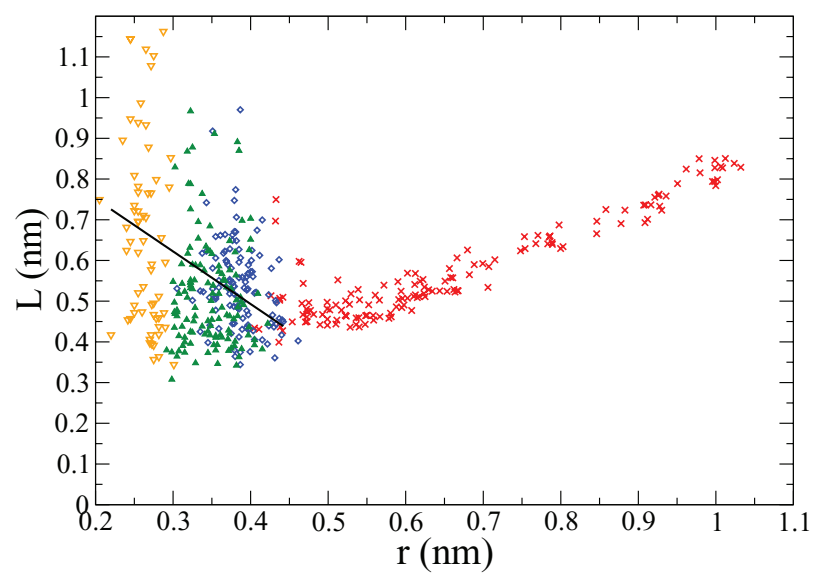

FIG. 11. Localization length, $L=\left(\left\langle r^{2}\right\rangle-\langle r\rangle^{2}\right)^{\frac{1}{2}}$, as a function of the average cavity radius for the same phases as in Fig. 10. The solid line is a linear fit of the data excluding the artificial cavities, used for the estimation of the number of extra cavities according to Eq. (5). 


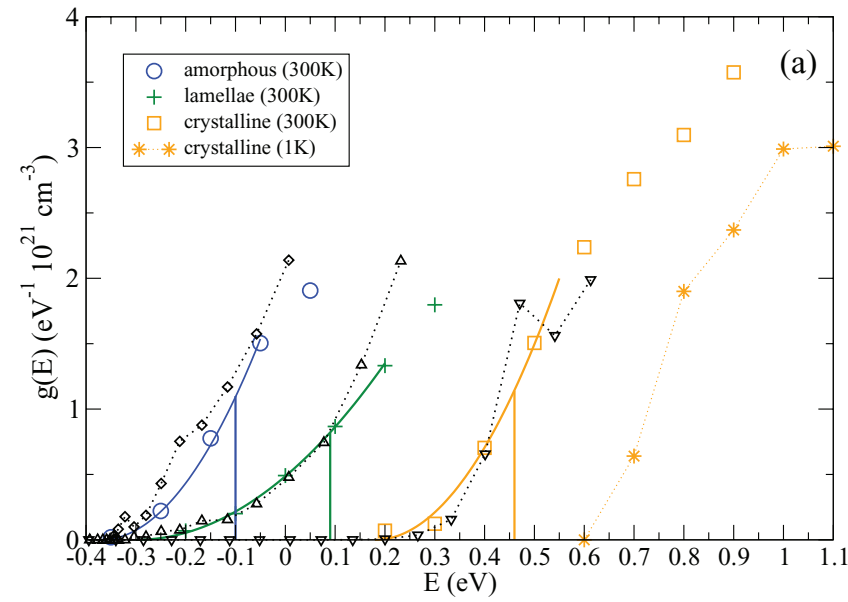

FIG. 12. Same as in Fig. 2(a) but with the filled symbols showing the prediction from the cavity density calculation. The dotted lines are a guide to the eye.

cavities with radii large enough that localized states can be associated with them, i.e., cavities with radii larger than $r_{c}$, the cavity radius associated with the mobility edge $E_{c}$. We are interested on the total number of extra cavities which are overcounted per cavity in the prediction of the number of localized states. A simple estimation is given by the formula

$$
\bar{N}=\frac{4 \pi}{3}\left({\overline{r_{L}}}^{3}-\bar{r}^{3}\right) \int_{r_{c}}^{\infty} f(r) d r,
$$

where $\quad \overline{r_{L}}=\int_{r_{c}}^{\infty} r_{L}(r) f(r) d r / \int_{r_{c}}^{\infty} f(r) d r \quad$ and $\quad \bar{r}$ $=\int_{r_{c}}^{\infty} r f(r) d r / \int_{r_{c}}^{\infty} f(r) d r$ are averages over the cavity distribution. The estimated density of states can then be corrected by dividing by the average number of cavities associated with each state, i.e., dividing the predicted density of states by $1+\bar{N}$. Using the linear fits indicated by solid lines in Figs. 9-11, we obtained $\bar{N}=0.93,1.4$, and 28.4 in the amorphous, lamellar, and crystalline samples, respectively. The average localization lengths in each Cartesian direction show a clear anisotropy in the crystalline samples. With the axes chosen with the $z$-axis running parallel to the PE chains in this phase, we obtained $\bar{L}_{x}=0.29 \mathrm{~nm}, \bar{L}_{y}=0.40 \mathrm{~nm}$, and $\bar{L}_{z}=0.51 \mathrm{~nm}$. However, the resulting correction, $\bar{N}=33.6$ is similar to the value obtained assuming an isotropic symmetry. The calculation involves replacing the spherical electronic volume in Eq. (5) by its Cartesian counterpart, $4 \pi \bar{r}_{L}^{3} / 3 \rightarrow 8 \bar{L}_{x} \bar{L}_{y} \bar{L}_{z} / 0.36^{3}$ (the factor 0.36 comes from the infinite square well model in Cartesian coordinates).

The resulting curves are shown in Fig. 12. Very good agreement is found between the direct count and the prediction from the density of states, even in the crystalline case, where the geometry is not isotropic. This is surprising, given the amount of scatter in the linear fit of Fig. 11 and the approximate nature of Eq. (5).

\section{DISCUSSION AND CONCLUSION}

Our simulations of the behaviour of an excess electron in the various structural motifs of polyethylene show that at room temperature the electron finds a rapidly rising density of states with a mobility edge separating localised from delocalised states. As more disorder is introduced into the structure (say from crystal to amorphous), the ground state moves from $+0.18 \mathrm{eV}$ to $-0.37 \mathrm{eV}$, with a proportionate reduction in the energy of the mobility edge. The density of states of the interfacial phases (lamellae-amorphous perpendicular and the lamellae-amorphous parallel) can be predicted by taking the DoS of each bulk phase and multiplying by the volume fraction of each phase present. Moreover, the lamella phase DoS, below the mobility edge, can itself by approximated by multiplying the inter-lamella volume fraction by the amorphous density of states so that a knowledge of the just the crystalline and amorphous density of states is sufficient to reproduce the DoS of all the interfacial phases. This suggests that the density of states for a heterogeneous material can be approximated by summing the single phase DoS weighted by their volume fractions in the material.

Another striking result is the low mobility of the electron in the lamella phase compared to the bulk amorphous and crystalline regions, which we can understand physically as arising from confining the relevant electron states to a small inter lamella region of the total volume. Although not calculated here, we would expect a similar low mobility for the other interfacial phases.

Furthermore, by associating the localized electron states with one or more sub nanometer cavities in polyethylene we are able to predict the density of states in a given motif, creating for the first time a quantitative link between the local properties of the polymeric material and the localized states of an excess electron.

Finally, let us note that the simulations reported here ignore polaronic effects, ${ }^{11}$ i.e., the distortion of the molecular configuration due to the presence of the excess electron. This does not affect our mobility calculations, since they are based on conduction though extended states, where the electron propagates fast enough so that the distortion of the (slow) molecules can be neglected and the delocalization of the electron in these states results in weak distorting forces on the nuclear framework. This is not the case for localized states, as shown in Ref. 11 for an excess electron in bulk amorphous PE, where the ground-state energy and localization length were observed to decrease by a small amount (of roughly about $0.1 \mathrm{eV}$ and $0.2 \mathrm{~nm}$, respectively) as the electron self-traps itself. This is not relevant, however, to the description of the (mainly excited) states available to excess electrons before they actually occupy them, and their relationship with the local properties of the polymeric material. But it remains to be seen how polaronic effects affect the dynamics of the electron at the interfaces. This is the subject of on-going investigation.

\section{ACKNOWLEDGMENTS}

D.F.C. acknowledges support from the US National Science Foundation under grant CHE-0911635 and from his Stokes Professorship in Nano Biophysics from Science Foundation Ireland. D.M. thanks the Irish Centre for High End Computing (ICHEC) for computer resources and Science Foundation Ireland for support from grant 08-IN.1-I1869. 
D.C. thanks support from State Key Lab. of Electrical Insulation and Power Equipment in Xian Jiaotong University under project EIPE13203.

${ }^{1}$ M. A. Henderson, J. M. White, H. Uetsuka, and H. Onishi, J. Am. Chem. Soc. 125, 14974 (2003).

${ }^{2}$ C. J. Lombardo, M. S. Glaz, Z.-E. Ooi, D. A. Vanden Bout, and A. Dodabalapur, Phys. Chem. Chem. Phys. 14, 13199 (2012).

${ }^{3}$ C. Groves, J. C. Blakesley, and N. C. Greenham, Nano Lett. 10, 1063 (2010).

${ }^{4}$ L. G. Kaake, P. F. Barbara, and X. Y. Zhu, J. Phys. Chem. Lett. 1, 628 (2010).

${ }^{5}$ G. Mazzanti, G. C. Montanari and J. M. Alison, IEEE Trans. Dielectr. Insul. 10, 187 (2003).

${ }^{6}$ P. F. Barbara, T. J. Meyer, and M. A. Ratner, J. Phys. Chem. 100, 13148 (1996).

${ }^{7}$ H. T. Nicolai, M. Kuik, G. A. H. Wetzelaer, B. de Boer, C. Campbell, C. Risko, J. L. Brédas, and P. W. M. Blom, Nature Mater. 11, 882 (2012).

${ }^{8}$ The first polyethylene insulated cable was a mile of submarine cable between the Isle of Wight and the mainland of England installed by Dean's company in 1938, see J. A. Allen, Studies in Innovation in the Steel and Chemical Industries (Manchester University Press, Manchester, 1967).

${ }^{9}$ M. Meunier and N. Quirke, J. Chem. Phys. 113, 369 (2000); M. Meunier, A. Aslanides, and N. Quirke, ibid. 115, 2876 (2001); G. Teyssedre, C. Laurent, A. Aslanides, N. Quirke, L. A. Dissado, A. Campus, and L. Martinotto, IEEE Trans. Dielectr. Electr. Insul. 8, 744 (2001).

${ }^{10}$ J. A. Anta, G. Marcelli, M. Meunier, and N. Quirke, J. Appl. Phys. 92, 1002 (2002).
${ }^{11}$ D. Cubero, N. Quirke, and D. F. Coker, J. Chem. Phys. 119, 2669 (2003); D. Cubero, D. F. Coker, and N. Quirke, Chem. Phys. Lett. 370, 21 (2003); D. Cubero and N. Quirke, J. Chem. Phys. 120, 7772 (2004).

${ }^{12}$ G. Dlubek, T. Lupke, H. M. Fretwell, M. A. Alam, A. Wutzler, and H. J. Radusch, Acta Polym. 49, 236 (1998).

${ }^{13}$ G. Dlubek, K. Saarinen, and H. M. Fretwell, J. Polym. Sci. B 36, 1513 (1998).

${ }^{14}$ H. A. Illias, G. Chen, and P. L. Lewin, J. Phys. D 44, 245202 (2011).

${ }^{15}$ See supplementary material at http://dx.doi.org/10.1063/1.4869831 for a detailed approach of the modeling of different morphologies of PE.

${ }^{16}$ L. A. Dissado and J. C. Fothergill, Electrical Degradation and Breakdown in Polymers (Peter Peregrinus, London, 1992).

${ }^{17}$ I. L. Hosier, A. S. Vaughan, and S. G. Swingler, J. Polym. Sci. B 38, 2309 (2000).

${ }^{18}$ D. C. Bassett, Principles of Polymer Morphology (Cambridge University Press, New York, 1981).

${ }^{19}$ D. C. Bassett, A. Keller, and S. Mitsuhasi, J. Polym. Sci. A 1, 763 (1963).

${ }^{20}$ B. Smit, S. Karaborni, and J. I. Siepmann, J. Chem. Phys. 102, 2126 (1995).

${ }^{21}$ J. Brandrup and E. H. Immergut, Polymer Handbook (American Institute of Physics, New York, 1989).

${ }^{22}$ N. Mullin and J. K. Hobbs, Phys. Rev. Lett. 107, 197801 (2011).

${ }^{23} \mathrm{See}$ http://www.surface-tension.de/solid-surface-energy.htm for information about the surface tension data of linear PE.

${ }^{24}$ F. Webster, P. J. Rossky, and R. A. Friesner, Comput. Phys. Commun. 63, 494 (1991); L. Turi and P. J. Rossky, Chem. Rev. 112, 5641 (2012).

${ }^{25}$ N. Ueno, K. Sugita, K. Seki, and H. Inokuchi, Phys. Rev. B 34, 6386 (1986).

${ }^{26}$ S. Serra, S. Iarlori, E. Tosatti, S. Scandolo, M. C. Righi, and G. E. Santoro, Chem. Phys. Lett. 360, 487 (2002). 